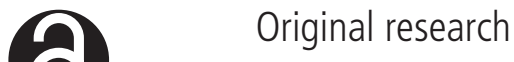

\section{Embotrap Extraction \& Clot Evaluation \& Lesion Evaluation for NeuroThrombectomy (EXCELLENT) Registry design and methods}

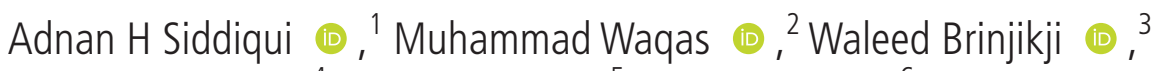 \\ Simon F De Meyer, ${ }^{4}$ Karen Doyle (1) ${ }_{1}^{5}$ Jens Fiehler (10 , ${ }^{6}$ Werner Hacke, ${ }^{7}$ \\ Ricardo A Hanel (1) , ${ }^{8}$ Tudor G Jovin, ${ }^{9}$ David S Liebeskind, ${ }^{10}$ Albert J Yoo, ${ }^{11}$ \\ Osama 0 Zaidat ${ }^{10},{ }^{12}$ Tommy Andersson, ${ }^{13,14}$ Raul G Nogueira ${ }^{15}$
}

\begin{abstract}
- Additional supplemental material is published online only. To view, please visit the journal online (http://dx.doi. org/10.1136/neurintsurg2021-017671)
\end{abstract}

For numbered affiliations see end of article.

Correspondence to Dr Adnan H Siddiqui, University at Buffalo Jacobs School of Medicine and Biomedical Sciences, Buffalo, New York, USA; asiddiqui@ubns.com

AHS and RGN contributed equally.

Received 27 April 2021 Accepted 8 August 202

Check for updates

(c) Author(s) (or their employer(s)) 2021. Re-use permitted under CC BY-NC. No commercial re-use. See rights and permissions. Published by BMJ.

To cite: Siddiqui $\mathrm{AH}$ Waqas M, Brinjikji W, et al. J Neurolntervent Surg Epub ahead of print: [please include Day Month Year]. doi:10.1136/

neurintsurg-2021-017671

\section{ABSTRACT}

Background Relationships between occlusive clot histopathology, baseline characteristics, imaging findings, revascularization rates, and clinical outcomes of stroke patients with large vessel occlusion (LVO) are not well understood. This study will assess the real-world experience on the efficacy and safety of using the EmboTrap device as the first approach in LVO patients and explore the associations between clot histological characteristics, imaging and clinical findings, revascularization rates, and clinical outcomes.

Methods Prospective, global, multicenter, singlearm, imaging core laboratory, and clot analysis central laboratory observational registry. Adult patients (>18 years) with LVO, treated with EmboTrap as the first attempted device, will be eligible for study participation. Results Up to 1000 subjects at 50 international sites may be enrolled. Occlusive clots will be collected from at least 500 subjects. Independent central and imaging core laboratories will perform clot analysis and image adjudication. Statistical analysis will assess the association between imaging and clinical findings, clot characteristics, subject comorbidities, revascularization, and clinical outcomes. Study endpoints are functional independence (modified Rankin Scale score $\leq 2$ at 90 days), expanded Thrombolysis In Cerebral Infarction (eTICI) score $\geq 2$ b50 rate, first-pass effect, number of passes, embolization into new territory, symptomatic intracranial hemorrhage, and 90-day mortality.

Conclusions The EXCELLENT registry will provide reproducible effectiveness and safety data of EmboTrap for its use for mechanical thrombectomy. Additionally, the study will characterize the blood clots retrieved during mechanical thrombectomy with respect to their composition and histopathological analysis and potential correlations with clinical and imaging findings. Trial registration number NCT03685578.

\section{BACKGROUND}

According to the World Health Organization, 15 million people suffer from a stroke per year, leaving 5 million dead and 5 million severely disabled. ${ }^{1}$ Approximately $90 \%$ of all strokes are acute ischemic strokes (AIS). Estimates on the percentage of AIS due to large vessel occlusion (LVO)_internal carotid artery, middle cerebral artery, vertebral artery, and basilar artery-vary from $15.8 \%$ to $46 \%$ depending on the definition methodology and nature of the screened population. ${ }^{2}$ Meta-analyses of randomized controlled trials successfully demonstrated the safety and effectiveness of mechanical thrombectomy (MT) compared with medical therapy alone in treating AIS in patients secondary to LVOs. ${ }^{3}$ As a result, treatment guidelines recommended MT as the standard of care. ${ }^{4}$

Trials included in the HERMES pooled analysis used stent retrievers such as Trevo and Solitaire FR. While these devices were safe and effective in the treatment of AIS, successful revascularization was not achieved in a subset of patients. ${ }^{56}$ The SEERS and HERMES pooled analysis of all randomized controlled trials reported a revascularization rate (modified Thrombolysis In Cerebral Infarction (mTICI) 2b/3) of 71\%. ${ }^{5}$ However, complete reperfusion (Thrombolysis In Cerebral Infarction (TICI) 3 ) on first pass was achieved in only 33\%. It is hypothesized that anatomical variations and clot characteristics are contributing factors to achieving complete revascularization, underscoring the need for additional advances in MT devices. ${ }^{7}$ Therefore, it is important to evaluate predictors of failed revascularization.

The EmboTrap Revascularization Device (Neuravi/Cerenovus) was designed specifically to perform across a range of clots with varying characteristics in the anterior and posterior neurovasculature. EmboTrap is delivered, unsheathed, and deployed in a fashion similar to other MT devices on the market. It traps the clot within the petals, thus allowing successful retrieval of the clot and restoration of blood flow. ${ }^{8}$

Previous international, multicenter clinical trials on the use of the EmboTrap Revascularization Device, that is, ARISE and ARISE II, showed impressive rates of final successful revascularization (mTICI 2b-3) of 95\% and 93\%, respectively. In the ARISE II study, $80 \%$ of the subjects enrolled achieved successful revascularization (expanded Thrombolysis In Cerebral Infarction (eTICI) $\geq 2$ b50) within three device passes. Overall, $67 \%$ of the subjects in ARISE II achieved a modified Rankin Scale (mRS) score of 0-2 at 90 days. ${ }^{9}$ 


\section{Box 1 Inclusion and exclusion criteria}

\section{Inclusion criteria:}

Candidates for this study must meet ALL of the following criteria:

- Age $\geq 18$ years

- Informed consent

- Subjects experiencing AIS with angiographic confirmation of LVO

- A clinical decision made to use the EmboTrap Revascularization Device independently and before enrollment in the research study

- EmboTrap Revascularization Device is the first attempted device/technique for MT in the subject.

Exclusion criteria:

Subjects will be excluded if ANY of the following apply:

- Participation in a clinical trial that may confound study endpoints

- Positive pregnancy test.

Subgroup comparable to previous studies utilizing MT: A subgroup of subjects will be identified through pre-specified eligibility criteria consistent with studies utilized for regulatory clearance of thrombectomy devices in the USA.

- $\mathrm{mRS} \leq 1$

- NIHSS $\geq 8$ and $<30$

- Occlusion location=ICA, M1 or M2

- ASPECTS $\geq 6$

- Subjects treated within 6 hours hours of onset of AIS

- Subjects who did not receive IV thrombolysis or received it within 3 hours of AIS onset

- Subjects that did not undergo carotid stenting or angioplasty.

AIS, acute ischemic stroke; ASPECTS, Alberta Stroke Program Early CT Score; ICA, internal carotid artery; IV thrombolysis, intravenous tissue plasminogen activator; LVO, large vessel occlusion; mRS, modified Rankin Scale; MT, mechanical thrombectomy; NIHSS, National Institutes of Health Stroke Scale.

To demonstrate the reproducibility of these results, the Embotrap Extraction \& Clot Evaluation \& Lesion Evaluation for NeuroThrombectomy (EXCELLENT) Real-world International Registry was initiated. The primary objective of the study is to evaluate the efficacy and safety of the EmboTrap revascularization device in a phase IV post-marketing real-world large prospective registry. The secondary objective is to explore the associations between baseline imaging characteristics, clot histology, and subject comorbidities with clinical and revascularization outcomes. Here we present the methodology of the EXCELLENT registry.

\section{METHODS/DESIGN}

\section{Design}

This is a prospective, global, multicenter, single-arm, observational registry. Per agreement with the Food and Drug Administration (FDA), the registry will be conducted under an investigational device exemption. The registry will enroll patients at 50 sites in the USA, Europe, and other regions (participating sites are listed in online supplemental table 1).

\section{Patient population: inclusion and exclusion criteria}

Patients with angiographically confirmed LVO, undergoing MT with EmboTrap as the first attempt device for the intracranial
Baseline: Screening / ICF, Medical History, IV t-PA (as applicable), Imaging, NIHSS, pre-stroke mRS

Procedure: Screening / ICF, angiographic confirmation of LVO and the EmboTrap ${ }^{\circledR}$ Revascularization Device as the first attempted device, per pass clot collection (as applicable), Review AEs

24 hours Post Procedure (-12/+48hours): ICF, Imaging, NIHSS, Review AES

Discharge or 7 days $(-1 /+7$ days): ICF, NIHSS, mRS, Review AEs

Subject

Follow-up

90 Day Follow-up (+/- 15days): mRS, Review AEs

Figure 1 Schematic of study design — flow chart. AEs, adverse events; ICF, informed consent form; IV-tPA, intravenous tissue plasminogen activator; LVO, large vessel occlusion; MRS, modified Rankin Scale; NIHSS, National Institutes of Health Stroke Scale.

occlusion, will be included in the study. Full and detailed eligibility criteria are presented in box 1 .

\section{Intervention}

Eligible patients will be enrolled after signing an informed consent in accordance with local regulations and institutional review board/ethics committee (IRB/EC) protocol (IRB/EC approval information is provided in online supplemental table 1). Due to the emergent nature of AIS, if approved by IRB/ EC consent may be obtained after the procedure but prior to 7 days or if not approved consent will be obtained pre-procedure (study flow chart is outlined in figure 1). The enrolled subject will undergo endovascular MT with the EmboTrap Revascularization Device as the first attempted treatment device for the intracranial occlusion. The minimum required number of MT passes with EmboTrap is one. The treating interventionist may decide to use any other device or technique if reperfusion is not achieved with EmboTrap. Thrombectomy devices (eg, use of stent retriever, aspiration, balloon guide, intermediate catheter, long sheath catheter) along with technique details (eg, incubation time, device positioning, co-aspiration), as well as rates and reasons for device changes, will be captured for each procedural pass.

Clot collection and analysis at independent central laboratory Blood clot retrieved during each pass of the stent retriever will be collected at sites with infrastructure for clot collection and transfer (online supplemental table 1). Clots will be collected from a minimum of 500 subjects. Clots retrieved with each pass will be collected separately in neutral buffered formalin solution and de-identified for transport. Composition and histopathological analysis of clots will be performed at an independent central laboratory. Relative content of red blood cells (RBC), white blood cells, platelets, fibrin and other fibrous proteins will be evaluated by experts in the laboratory. Successful achievement of this endpoint will facilitate analysis of effective removal of clots that span the range from RBC-poor $(<25 \%)$ to RBC-rich $(>75 \%)$. Hematoxylin and eosin (H\&E) staining will also be conducted and scans made available for analysis. 


\begin{tabular}{|c|c|c|}
\hline \multicolumn{2}{|c|}{ Independent Imaging Core Lab } & $\begin{array}{l}\text { Independent } \\
\text { Central Lab }\end{array}$ \\
\hline $\begin{array}{l}\text { Performance Evaluation } \\
\begin{array}{l}>\text { Successful Revascularization } \\
>\text { First Pass Effect } \\
>\text { Modified First Pass Effect } \\
>\text { Procedure Time - time from } \\
\text { groin puncture to } \\
\text { revascularization } \\
>\text { Occurrence of Embolization } \\
\text { to a New Territory }\end{array}\end{array}$ & $\begin{array}{l}\text { Imaging Analysis } \\
>\text { Ischemic } \\
\text { Core/Penumbra } \\
\text { volume analysis } \\
>\text { Post-procedure } \\
\text { ICH }\end{array}$ & $\begin{array}{l}\text { Clot Analysis } \\
>\text { Clot composition } \\
>\text { Histopathologic } \\
\text { analysis }\end{array}$ \\
\hline
\end{tabular}

Figure 2 Role of independent imaging core laboratory and central laboratory. ICH, intracerebral hemorrhage.

\section{Independent core imaging laboratory}

An independent core laboratory will perform blinded assessments of imaging parameters at entry, and the assessments for the primary and secondary imaging endpoints, including eTICI, infarct volume, intracerebral hemorrhage $(\mathrm{ICH})$ type along with emboli in new territory. These evaluations will be performed by experienced independent readers. Figure 2 illustrates the role of the independent core laboratory.

\section{Functional outcomes}

Functional independence will be assessed by mRS $(\mathrm{mRS} \leq 2)$ at $90 \pm 15$ days performed by a qualified evaluator independent of the interventional treating team

\section{Clinical efficacy}

Clinical efficacy outcomes of the study are: (1) $\mathrm{mRS} 0-2$ at 90 days or equal to pre-stroke mRS value; (2) change in the National Institutes of Health Stroke Scale (NIHSS) score at 24 hours compared with baseline; (3) death due to any cause at 90 days.

Performance outcome will be successful revascularization with final eTICI $\geq 2 \mathrm{~b} 50 .{ }^{10} 11$ Other performance parameters will include final revascularization with eTICI $\geq 2 c$, first pass effect (eTICI $\geq 2 \mathrm{c}$ after the first pass without rescue), modified first pass effect (eTICI $\geq 2 \mathrm{~b} 50$ after the first pass), procedure time defined as time from groin puncture to the time of first achievement of eTICI score $\geq 2 b$, as long as the final (end of procedure) eTICI score is not worse than $2 b$, number of passes and occurrence of embolization into a new territory. Any change of therapy or frontline device to treat the target lesion before achieving revascularization (eTICI $\geq 2 b$ ) is considered rescue therapy.

Safety endpoints will include symtomatic ICH at 24 hours specified according to the Heidelberg Bleeding Classification $^{11}$ — defined as a new ICH as detected by brain imaging after 24 hours of intervention associated with:

- $\geq 4$ points worsening of total NIHSS score at the time of diagnosis compared with immediately before deterioration

- $\geq 2$ point worsening in one NIHSS category

- Leading to intubation/hemicraniectomy/external ventricular drain placement or other major medical/surgical intervention

- Absence of alternative explanation for deterioration.

Other safety outcomes will include all strokes, device and/or procedure related adverse events, vessel perforation, dissection or injury, vascular access injury, and neurologic deterioration (change in NIHSS $\geq 4$ points from last known) through 7 days/ discharge, whichever comes first.

\section{Data monitoring body}

The study will be conducted in accordance with Good Clinical Practice, ISO 14155 requirements, local regulations, and the principles of the Declaration of Helsinki. Clinical site monitoring will ensure that the rights and well-being of human subjects are protected, that study data are accurate, complete, and verifiable, and that study conduct is in compliance with the aforementioned regulations.

Data are to be submitted via electronic case report forms after collection. Missing or unclear data will be queried to be corrected as necessary throughout the study. Additional clinical documentation may be requested to understand an adverse event.

\section{Sample size estimates}

Up to 1000 subjects will be enrolled, in keeping with the therapeutic area standards for similar post-market registries. Clot collection is expected to be achieved in a minimum of 500 subjects, based on projected enrollment at the contributing sites.

The sample size for a subgroup analysis has also been specified. A minimum of 184 consecutive subjects enrolled into this study will be identified through pre-specified eligibility criteria in order to define a cohort similar with regard to pre-stroke mRS, baseline NIHSS and clot location to those enrolled in prior MT studies (defined as the mRS sub-population). Based on the ARISE II study outcomes and the real-world setting for the study, the anticipated proportion of subjects reaching mRS score 0-2 at day 90 ranges between 53-55\%. Approximately 184 subjects will be needed to obtain at least $80 \%$ power to compare the proportion of subjects with $\mathrm{mRS}$ score $0-2$ at day 90 to a performance goal of $41.3 \%$ (established from a meta-analysis of MR CLEAN, TREVO 2, SWIFT and SWIFT PRIME) at a one-sided $\alpha$ of $2.5 \%$, using the exact method. ${ }^{6} 1213$ The assumed attrition is $20 \%$ to account for subjects who are lost to follow-up, early withdrawal or have missing outcomes.

\section{Statistical analyses}

Statistical analysis will be performed using SAS version 9.4.

Standard descriptive summaries for continuous data include the number of subjects and events with non-missing outcome, mean, standard deviation, median, minimum, and maximum values. For categorical data, the count and percentage will be provided. All demographic characteristics, procedural, imaging, postoperative data, and clot data will be summarized in the safety analysis population (all patients in whom the treatment is attempted) and modified intent to treat population (all patients who received treatment).

When the study is completed, the following analysis will be performed for the mRS sub-population. The number and percentage of subjects reaching mRS score $0-2$ at day 90 will be summarized. The exact two-sided $95 \%$ confidence intervals will be constructed around the percentage and the lower bound of the confidence intervals will be compared with the performance goal of $41.3 \%$. Missing mRS scores at day 90 will be handled with the use of the last-observation-carried-forward approach when a score is available from a post-procedure visit (eg, 7 days/ discharge or unscheduled visit). Live subjects who are missing any $\mathrm{mRS}$ scores post-baseline will be excluded from the analysis.

The $\chi^{2}$ test will be used when the expected cell frequency is $>5$ (or Fisher's exact test otherwise) for categorical variables and the t-test when the normality assumption is met (or other 
non-parametric tests as appropriate, for example, the MannWhitney U test) for comparison of numerical variables.

A periodic analysis (eg, annual, or more frequently based on enrollment) is planned to report data on the endpoints descriptively which may also be used for presentations at scientific meetings. Exploratory analyses will be performed in the clot population between: (1) baseline subject comorbidity and clot characteristics (clot characteristics as dependent variables); (2) clot characteristics and reperfusion (reperfusion as the dependent variable); and (3) baseline subject comorbidities, clot characteristics and reperfusion with clinical outcomes (clinical outcomes as dependent variables).

\section{Measures to minimize bias}

In addition to standardized independent central clot and core imaging laboratories, the following measures are planned to minimize bias:

1. An independent clinical events committee will review all safety endpoints and other adverse events of interest including all 24-hour ICHs

2. Neurological status and clinical outcomes will be assessed by certified evaluators using NIHSS and mRS which are standardized objective tools widely used in neurological research

3. The 90-day mRS will be performed by a qualified independent evaluator who is not part of the interventional treating team

4. Study monitors will have clinical research experience and be proficient at study monitoring. Study data will be source data verified using the subject's medical records, study source worksheets, clinic notes, and radiographic reports as applicable.

\section{Study organization and funding}

Study conduct will be overseen by the executive steering committee and the sponsor. The executive steering committee will be led by three global co-principal investigators. The steering committee will be comprised of experts in the field of stroke intervention and representatives of leading enrolling sites. Cerenovus will provide funding for the study.

\section{DISCUSSION}

The EXCELLENT registry will provide reproducible effectiveness and safety data of EmboTrap for its use for MT in a large prospective, global cohort of patients in real-world setting across different sites and operator experience levels. Additionally, the study will characterize the blood clots retrieved during MT with respect to their composition and histopathological analysis and potential correlations to clinical and imaging findings.

The composition of the clot determines its physical properties and may influence the rates of successful MT. ${ }^{14}$ Fibrin-rich clots are tough in consistency. ${ }^{14}$ In vitro MT models have indicated a high failure rate with existing stent retrievers for fibrinrich clots. ${ }^{15}$ Information on the composition and characteristics of clots retrieved during endovascular procedures is limited. EXCELLENT will provide a large repository of endovascularly obtained clots, intended to improve our understanding of clot properties and possible correlations to clinical, procedural, and imaging characteristics. Compared with other large repositories of thrombi retrieved in MT, this study additionally includes the collection of per pass device and technique details, use of an independent imaging core laboratory, and functional outcomes assessment by a qualified evaluator independent of the interventional treating team. The insights obtained will be used to improve the design of existing stent retrievers and refine the technique for MT.

The current study is designed to have a subgroup with predefined eligibility criteria, consistent with previous clinical trials on MT. This subgroup will be used to compare the results of EXCELLENT with studies used for recent clearances by regulatory agencies in the USA.

Another strength of the study is the use of independent central and imaging laboratories to evaluate the clot and imaging data from study subjects, and independent assessment of clinical outcomes. EXCELLENT will not restrict enrollment of patients currently being treated per standard of care, providing prospective real-world data on the effectiveness of EmboTrap in subjects receiving MT per the latest American Heart Association/ American Stroke Association (AHA/ASA) and European Stroke Organisation/European Society for Minimally Invasive Neurological Therapy (ESO/ESMINT) guidelines. ${ }^{416}$

Furthermore, the EXCELLENT real-life MT registry may include patients who are treated per routine care even though they do not fit the current guidelines, with good sample size to explore the efficacy and safety of EmboTrap in this population that may be harder to study in randomized clinical trials otherwise.

\section{CONCLUSIONS}

EXCELLENT is a prospective global registry designed to assess the effectiveness via real-world experience of the EmboTrap stent retriever and to explore the associations between imaging characteristics at presentation, clot characteristics, subject comorbidities, clinical outcomes, and revascularization rates.

\section{Author affiliations}

${ }^{1}$ University at Buffalo Jacobs School of Medicine and Biomedical Sciences, Buffalo, New York, USA

${ }^{2}$ Neurosurgery, University at Buffalo Jacobs School of Medicine and Biomedical Sciences, Buffalo, New York, USA

${ }^{3}$ Mayo Clinic Minnesota, Rochester, Minnesota, USA

${ }^{4}$ Laboratory for Thrombosis Research, KU Leuven, Campus Kulak Kortrijk, Kortrijk, Belgium

${ }^{5}$ Physiology, CURAM, National University of Ireland Galway, Galway, Ireland

${ }^{6}$ Dept. of Neuroradiology, University Medical Center Hamburg Eppendorf, Hamburg, Germany

${ }^{7}$ Neurology, University of Heidelberg, Heidelberg, Germany

${ }^{8}$ Lyerly Neurosurgery, Baptist Medical Center Downtown, Jacksonville, Florida, USA

${ }^{9}$ Neurology, Cooper University Hospital, Camden, New Jersey, USA

${ }^{10}$ Neurology, UCLA, Los Angeles, California, USA

${ }^{11}$ Neurointervention, Texas Stroke Institute, Plano, Texas, USA

${ }^{12}$ Neuroscience, St Vincent Mercy Hospital, Toledo, Ohio, USA

${ }^{13}$ Departments of Radiology and Neurology, AZ Groeninge, Kortrijk, Belgium

${ }^{14}$ Departments of Neuroradiology; Department of Clinical Neuroscience, Karolinska

University Hospital; Karolinska Institutet, Stockholm, Sweden

${ }^{15}$ Grady Memorial Hospital, Chickasha, Georgia, USA

Correction notice Since this article was published, the middle initial $\mathrm{F}$ has been added to the author name Simon De Meyer.

Twitter Jens Fiehler @Fie0815

Contributors AHS, WB, SDeM, KD, JF, WH, TGJ, DSL, AJY, OOZ, TA, and RGN conceptualized the study and developed the methodology. MW wrote the original draft of the protocol. AHS, TA and RGN provided study supervision. All authors reviewed, edited, and approved the final draft of the protocol.

Funding The EXCELLENT study is sponsored by Cerenovus (Irvine, California, USA).

Competing interests AHS: Co-investigator NIH/NINDS 1R01NS091075; Amnis

Therapeutics, Boston Scientific, Canon Medical Systems USA Inc, Cerebrotech Medical Systems, Cerenovus, Corindus Inc, Endostream Medical Ltd, Guidepoint Global Consulting, Imperative Care, Integra LifeSciences Corp, Medtronic,

MicroVention, Q'Apel Medical Inc, Rapid Medical, Rebound Therapeutics Corp, Serenity Medical Inc, Silk Road Medical, StimMed, Stryker, Three Rivers Medical, VasSol, W.L. Gore \& Associates; Amnis Therapeutics, Apama Medical, Blink TBI Inc, Buffalo Technology Partners Inc, Cardinal Consultants, Cerebrotech Medical Systems, 
Cognition Medical, Endostream Medical Ltd, Imperative Care, International Medical Distribution Partners, Neurovascular Diagnostics Inc, Q'Apel Medical Inc, Rebound Therapeutics Corp, Rist Neurovascular Inc, Serenity Medical, Silk Road Medical, StimMed, Synchron, Three Rivers Medical Inc, Viseon Spine. TA: Neuravi, Ablynx, Amnis Therapeutics, Medtronic, Rapid Medical, Stryker. RGN: Neuravi, Medtronic, Penumbra, Stryker.

Patient consent for publication Not required.

Provenance and peer review Not commissioned; externally peer reviewed.

Data availability statement Data sharing not applicable as no datasets generated and/or analyzed for this study.

Supplemental material This content has been supplied by the author(s). It has not been vetted by BMJ Publishing Group Limited (BMJ) and may not have been peer-reviewed. Any opinions or recommendations discussed are solely those of the author(s) and are not endorsed by BMJ. BMJ disclaims all liability and responsibility arising from any reliance placed on the content. Where the content includes any translated material, BMJ does not warrant the accuracy and reliability of the translations (including but not limited to local regulations, clinical guidelines, terminology, drug names and drug dosages), and is not responsible for any error and/or omissions arising from translation and adaptation or otherwise.

Open access This is an open access article distributed in accordance with the Creative Commons Attribution Non Commercial (CC BY-NC 4.0) license, which permits others to distribute, remix, adapt, build upon this work non-commercially, and license their derivative works on different terms, provided the original work is properly cited, appropriate credit is given, any changes made indicated, and the use is non-commercial. See: http://creativecommons.org/licenses/by-nc/4.0/.

\section{ORCID iDs}

Adnan H Siddiqui http://orcid.org/0000-0002-9519-0059

Muhammad Wagas http://orcid.org/0000-0003-4500-7954

Waleed Brinjikji http://orcid.org/0000-0001-5271-5524

Karen Doyle http://orcid.org/0000-0001-8984-6296

Jens Fiehler http://orcid.org/0000-0001-8533-7478

Ricardo A Hanel http://orcid.org/0000-0001-7195-5806

Osama O Zaidat http://orcid.org/0000-0003-4881-4698

\section{REFERENCES}

1 World Health Organization. The world health report: reducing risks, promoting healthy life.; 2002.
2 Lakomkin N, Dhamoon M, Carroll K, et al. Prevalence of large vessel occlusion in patients presenting with acute ischemic stroke: a 10-year systematic review of the literature. J Neurointerv Surg 2019;11:241-5.

3 Goyal M, Menon BK, van Zwam WH, et al. Endovascular thrombectomy after large-vessel ischaemic stroke: a meta-analysis of individual patient data from five randomised trials. Lancet 2016;387:1723-31.

4 Powers WJ, Rabinstein AA, Ackerson T. Guidelines for the early management of patients with acute ischemic stroke: a guideline for healthcare professionals from the American Heart Association/American Stroke Association. Stroke 2018;2018:e46-110.

5 Campbell BCV, Hill MD, Rubiera M, et al. Safety and efficacy of solitaire stent thrombectomy: individual patient data meta-analysis of randomized trials. Stroke 2016;47:798-806.

6 Nogueira RG, Lutsep HL, Gupta R, et al. Trevo versus Merci retrievers for thrombectomy revascularisation of large vessel occlusions in acute ischaemic stroke (TREVO 2): a randomised trial. Lancet 2012;380:1231-40.

7 Duffy S, Farrell M, McArdle K, et al. Novel methodology to replicate clot analogs with diverse composition in acute ischemic stroke. J Neurointerv Surg 2017;9:486-91.

8 Chueh J-Y, Marosfoi MG, Brooks OW, et al. Novel distal emboli protection technology: the EmboTrap. Interv Neurol 2017;6:268-76.

9 Zaidat 00, Bozorgchami H, Ribó M, et al. Primary results of the multicenter ARISE II study (Analysis of Revascularization in Ischemic Stroke with EmboTrap). Stroke 2018;49:1107-15.

10 Liebeskind DS, Bracard S, Guillemin F, et al. eTICI reperfusion: defining success in endovascular stroke therapy. J Neurointerv Surg 2019;11:433-8.

11 Behme D, Tsogkas I, Colla R, et al. Validation of the extended thrombolysis in cerebral infarction score in a real world cohort. PLoS One 2019;14:e0210334.

12 Saver JL, Goyal M, Bonafe A, et al. Stent-retriever thrombectomy after intravenous t-PA vs. t-PA alone in stroke. N Eng/ J Med 2015;372:2285-95.

13 Saver JL, Jahan R, Levy El, et al. Solitaire flow restoration device versus the Merci retriever in patients with acute ischaemic stroke (SWIFT): a randomised, parallelgroup, non-inferiority trial. Lancet 2012;380:1241-9.

14 Yuki I, Kan I, Vinters HV, et al. The impact of thromboemboli histology on the performance of a mechanical thrombectomy device. AJNR Am J Neuroradiol 2012:33:643-8.

15 Fennell VS, Setlur Nagesh SV, Meess KM, et al. What to do about fibrin rich 'tough clots'? Comparing the Solitaire stent retriever with a novel geometric clot extractor in an in vitro stroke model. J Neurointerv Surg 2018;10:907-10.

16 Turc G, Bhogal P, Fischer U, et al. European Stroke Organisation (ESO)-European Society for Minimally Invasive Neurological Therapy (ESMINT) guidelines on mechanical thrombectomy in acute ischemic stroke. J Neurointerv Surg 2019;11:535-8. 